\title{
Imaging of Individual Anatomical Risk Factors for Patellar Instability
}

\author{
Tobias J. Dietrich, $M D^{1} \quad$ Sandro F. Fucentese, $M^{2}$ \\ ${ }^{1}$ Department of Radiology, Orthopedic University Hospital Balgrist,
Faculty of Medicine, University of Zurich, Zurich, Switzerland
2 Department of Orthopedic Surgery, Orthopedic University Hospital
Balgrist, Faculty of Medicine, University of Zurich, Zurich, Switzerland \\ Semin Musculoskelet Radiol 2016;20:65-73.
}

Christian W. A. Pfirrmann, MD, MBA ${ }^{1}$

Address for correspondence Tobias J. Dietrich, MD, Department of Radiology, Orthopedic University Hospital Balgrist, Forchstrasse 340, CH-8008 Zurich, Switzerland (e-mail: tobiasjdietrich@gmail.com).

\begin{abstract}
Keywords

- patellar instability

- risk factors

- trochlear dysplasia

- TTTG

- patellar tilt dislocation. tubercle.

Lateral dislocations of the patella are frequent injuries in sportsactive adolescents and young adults. ${ }^{1}$ The patellofemoral joint is stabilized by a complex multifactorial relationship of the osseous joint geometry and the force vectors resulting from the quadriceps muscle and capsuloligamentous stabilizers. ${ }^{2-4}$ For appropriate treatment planning, the radiologic work-up after an episode of lateral patellar dislocation should assess both the injuries of the knee and the individual anatomical risk factors for patellar instability. $1,3,5-7$
\end{abstract}

This review article presents several pitfalls and limitations of image interpretation of anatomical risk factors for patellar instability. The most important imaging examinations for the work-up of patients with patellar instability are the true lateral radiograph and transverse computed tomography (CT) or MR images of the knee. Primary anatomical risk factors are an insufficient medial patellofemoral ligament (MPFL), patella alta, trochlear dysplasia, increased distance from the tibial tuberosity to the trochlear groove (TTTG), and torsional limb parameters.

Limitations of the Caton-Deschamps index are related to the clear identification of the patellar and tibial articular margin. Classification of trochlear dysplasia according to the Dejour system on radiographs and MR images revealed a weak reliability. The comparability of TTTG values obtained on CT and MR images at various flexion angles and different varus alignments of the knee is limited. Thus MRI performed with a dedicated knee coil may underestimate the TTTG distance compared with CT images. Increased lateral patellar tilt is a consequence of primary anatomical risk factors rather than an independent anatomical risk factor for patellar instability. The pretest likelihood of a torn MPFL on MR images is very high after an acute episode of lateral patellar

Surgical restoration of the patellofemoral joint stability addresses the complex multifactorial biomechanics by a custom-made management such as MPFL reconstruction, sulcus-deepening trochleoplasty, as well as medialization and distalization of the tibial

Quantification of anatomical risk factors for patellar instability in each person is important for highly individual treatment.
Dejour et al provided reference values for imaging analysis of four major risk factors for patellar instability: patella alta, trochlear dysplasia, increased tuberosity to the trochlear groove (TTTG) distance, and abnormal lateral tilt of the patella. $3,6,8$ Additional anatomical risk factors for patellar instability are an insufficient medial patellofemoral ligament (MPFL), torsional limb malalignment, and genu valgum. ${ }^{5,9-11}$ Conventional radiographs, computed tomography (CT), and MRI enable quantification of these predisposing anatomical risk factors for patellar
Issue Theme Knee and Ankle Imaging; Guest Editor, Marco Zanetti, MD
Copyright (c) 2016 by Thieme Medical Publishers, Inc., 333 Seventh Avenue, New York, NY 10001, USA. Tel: +1(212) 584-4662.
DOI http://dx.doi.org/ 10.1055/s-0036-1579675. ISSN 1089-7860. 
instability. ${ }^{3,6,9-12}$ Surgical restoration of the patellofemoral joint stability addresses the complex multifactorial biomechanics by a tailored treatment such as MPFL reconstruction, release or lengthening of the lateral patellar retinaculum, medialization and distalization of the tibial tubercle, sulcus-deepening trochleoplasty, and rarely femoral derotation osteotomy. ${ }^{6-8,13,14}$ This review article presents several pitfalls and limitations in the analysis of anatomical risk factors for patellar instability.

\section{Patellar Height}

Patella alta, or high-riding patella, is a major risk factor for recurrent lateral patellar dislocations. ${ }^{3,8}$ The trochlear groove is the most important osseous stabilizer of the patellofemoral joint. ${ }^{1}$ The patella slips into the trochlear groove at $\sim 20$ degrees of knee flexion. ${ }^{1,15}$ A high-riding patella requires higher flexion angles of the knee to slide into the trochlear groove and covers a higher range of motion without osseous stabilization of the trochlear groove. ${ }^{1,5}$ Numerous quantification techniques on radiographs were proposed for the diagnosis of a high-riding patella. ${ }^{16-21}$ The four most popular patellar height measurement techniques are the Insall-Salvati, Grelsamer-Meadows, CatonDeschamps, and Blackburne-Peel indexes. ${ }^{21}$ Dejour and Le Coultre stated that any patellar height index can be used; however, the Caton-Deschamps index became the preferred standard for the Lyon school of knee surgery (-Fig. 1). ${ }^{6,8} \mathrm{~A}$

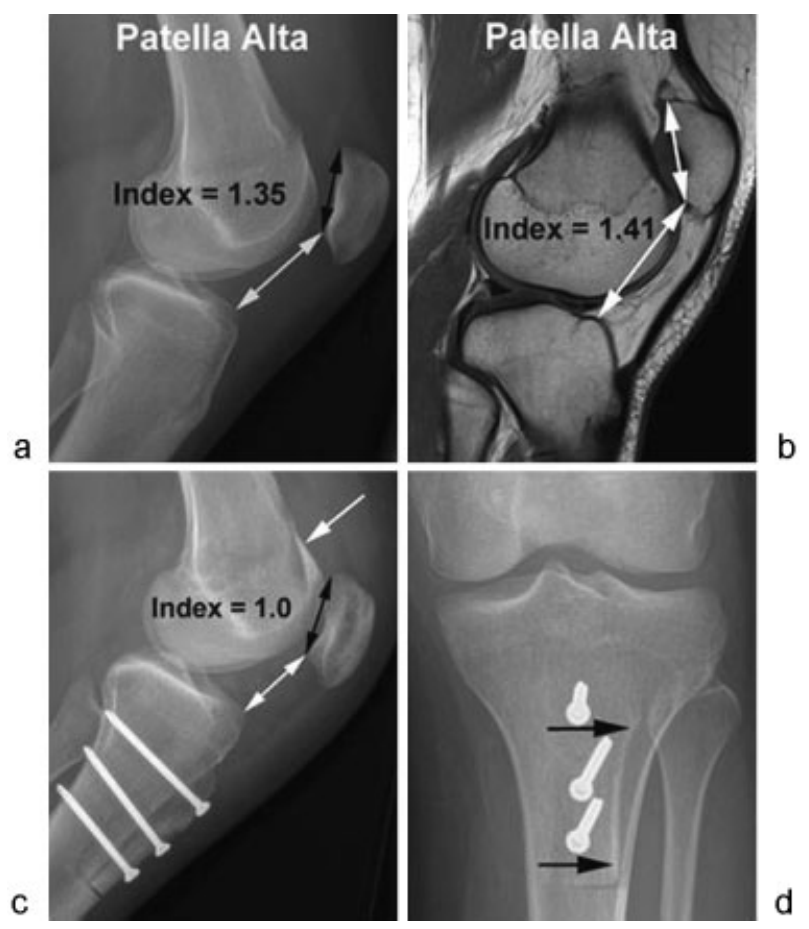

Fig. 1 (a) Caton-Deschamps index of 1.35 on the radiograph indicates a patella alta. (b) Corresponding Caton-Deschamps index was 1.41 on the sagittal intermediate-weighted MR image. Patellar height ratios on MR and computed tomography images are typically slightly higher than patellar height ratios on conventional radiographs (reference 25). (c, d) The 6-week postoperative follow-up radiographs show typical postoperative findings after sulcus-deepening trochleoplasty (arrow, c), medialization and distalization of the tibial tubercle (c, arrows, d). A clear reduction of the patellar height and Caton-Deschamps index was achieved. Medial patellofemoral ligament reconstruction was also performed on this knee. main advantage of the Caton-Deschamps and Blackburne-Peel ratios is the ability to quantify patellar height changes after osteotomy of the tibial tubercle (-Fig. 1). ${ }^{6}$ In addition, the Caton-Deschamps index allows simple and reliable patellar height measurements for various degrees of knee flexion, different physical sizes of the knee, variable skeletal maturation, patellar pole abnormalities, and unequal radiographic magnifications. $^{6,13,22,23}$ Limitations of the Caton-Deschamps index are related to the clear identification of the patellar and tibial articular margin as well as its difficult application in osteoarthritic knees. ${ }^{21}$ The Caton-Deschamps index is determined by the ratio of the distance between the lowest point of the patellar articular surface and the anterior point of the tibial plateau as well as the patellar articular length ( - Fig. 1 ), whereas values $>1.2$ indicates a patella alta and values $<0.6$ represent a patella baja. ${ }^{6,17}$ However the suggested reference values for patella alta and patella baja vary slightly in the literature.

The Insall-Salvati and Blackburne-Peel indexes on radiographs were compared with the corresponding values on CT and MR images. ${ }^{24,25} \mathrm{~A}$ moderate inter-method reliability of patellar height assessment was found for radiographs and CT images as well as for radiographs and MRI for both indexes. ${ }^{25}$ The results show that reference values for patella alta and baja based on radiographs cannot be directly applied on CT and MR images. ${ }^{3}$ The authors suggested the addition of 0.09 to 0.13 to the so far established cut-off values derived from conventional radiographs for the diagnosis of patella alta and baja on CT and MRI images. ${ }^{25}$

\section{Trochlear Dysplasia}

Trochlear dysplasia is considered a developmental anomaly and is one of the most important risk factors for lateral patellar dislocation. ${ }^{3,5}$ A decreased depth, a flat or even a convex trochlear surface characterizes trochlear dysplasia (-Figs. 2-6). . $^{3,8,9}$ Trochlea dysplasia can be diagnosed and classified according to the four types of David Dejour classification by the so-called crossing sign, a reference line along the anterior cortical border of the distal femur, the supratrochlear spur, and the double-contour sign on true lateral conventional radiographs, axial conventional radiographs,
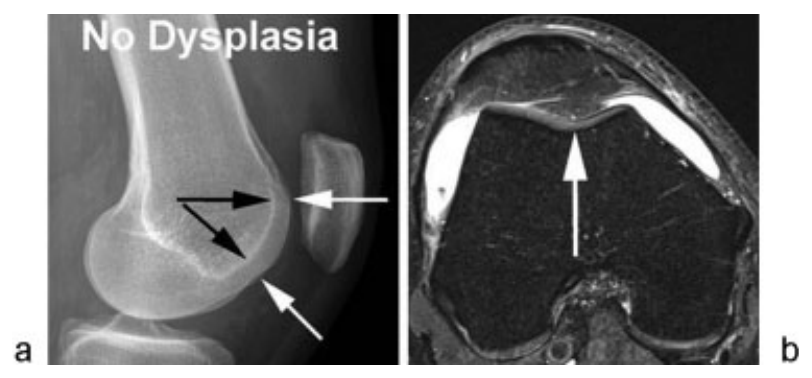

Fig. 2 Normal trochlea. (a) The radiopaque line on radiographs (black arrows) representing the sulcus of the femoral trochlea in a normal developed trochlea is located more posteriorly compared with the femoral condyles (white arrows) on true lateral radiographs. (b) The normal trochlear sulcus (white arrow) is concave on transverse crosssectional images (fat-suppressed intermediate-weighted MR image). 

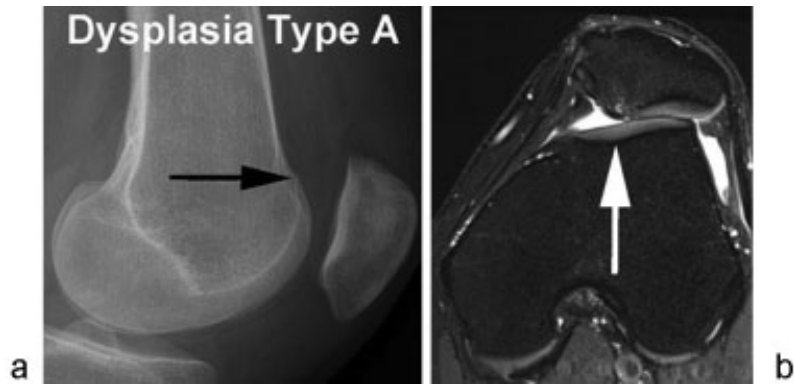

Fig. 3 Trochlear dysplasia type A according to Dejour. (a) Delineation of the crossing sign (arrow) on true lateral radiographs and (b) a shallow femoral trochlea (arrow) on transverse computed tomography or MR images (fat-suppressed intermediate-weighted MR image) defines trochlear dysplasia type A. Nevertheless, the trochlea should still be concave and symmetrical. ${ }^{8,9}$
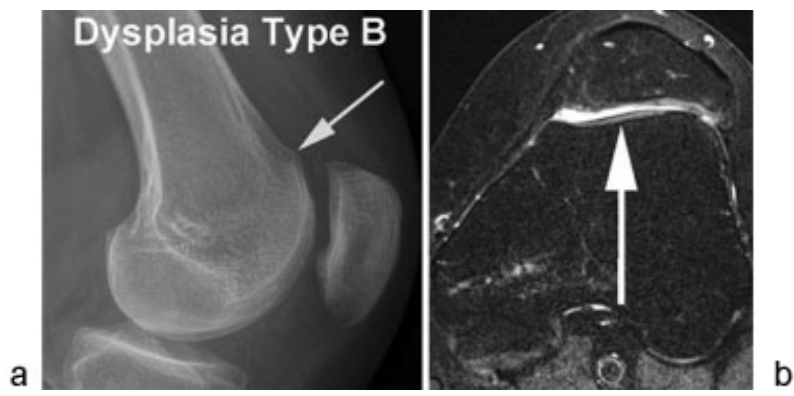

Fig. 4 Trochlear dysplasia type B according to Dejour. (a) The presence of the crossing sign and a supratrochlear spur on lateral radiographs (arrow) in combination with a (b) flat or convex trochlea defines trochlear dysplasia type B (arrow). ${ }^{8,9}$
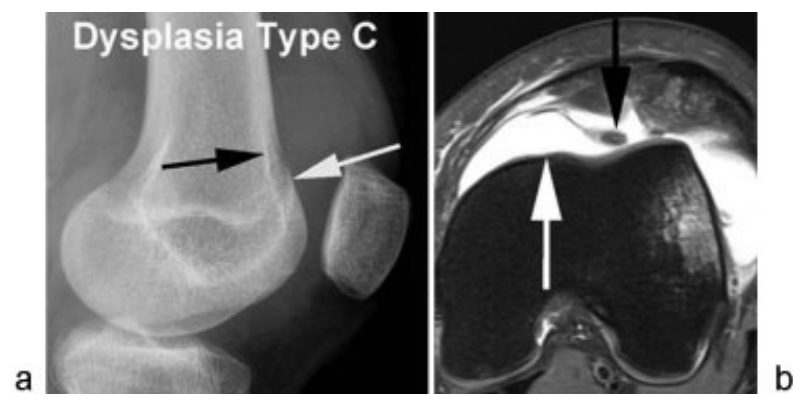

Fig. 5 Trochlear dysplasia type $C$ according to Dejour. (a) Coexistence of the crossing sign (white arrow), the double-contour sign (black arrow), absence of the supratrochlear spur on lateral radiographs, a convex lateral facet, and a hypoplastic medial facet (white arrow, b) represent type $C$ dysplasia. ${ }^{8,9}$ Black arrow on (b) points to an osseous avulsion of the medial patellofemoral ligament.

and midsagittal and transverse CT or MR images, respectively (-Figs. 2-6). ${ }^{3,8,9,26,27}$

The radiopaque line on radiographs representing the sulcus of the femoral trochlea in a normal developed trochlea is located more posteriorly compared with the femoral condyles on true lateral radiographs (-Fig. 2). ${ }^{8,9}$ A crossing sign defines trochlear dysplasia and is present if the line of the trochlear sulcus crosses the anterior outline of the femoral condyles on true lateral radiographs (-Figs. 3 and 5). ${ }^{8,9}$ The location of these crossing lines represents a flattened trochlea

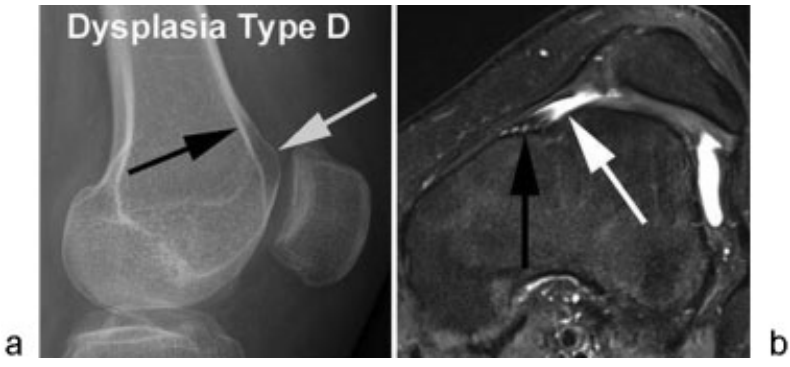

Fig. 6 Trochlear dysplasia type D according to Dejour. (a) The presence of the crossing sign, a supratrochlear spur (white arrow), the double-contour sign (black arrow) on lateral radiographs and a distinct asymmetry of the height of the medial (black arrow on b) and lateral trochlear facets, the so-called cliff-like pattern (white arrow on b) defines type D dysplasia. ${ }^{8,9}$

and indicates the shallowest part of the trochlear sulcus (-Figs. 3 and 5 ).

Two imaging findings differentiate between trochlear dysplasia Dejour type B, C and D. ${ }^{8,9}$ These findings are the supratrochlear spur (also called supratrochlear bump) and the double-contour sign (-Figs. 1, 6). ${ }^{8,9}$ The supratrochlear spur sign indicates an osseous prominence of the proximal part of the femoral trochlea, which is present if the height is $\geq 3 \mathrm{~mm}$ measured from a reference line along the anterior cortical border of the distal femur on true lateral radiographs. ${ }^{3}$ This reference line is usually anterior in relation to the sulcus of the trochlea in normal femoral trochlea $(-0.8 \pm 2.9 \mathrm{~mm}){ }^{3}$ The double-contour sign on lateral knee radiograph refers to a hypoplastic medial trochlear facet that represents a pronounced asymmetry of the height of the medial and lateral trochlear facets (-Figs. 5 and $\mathbf{6}$ ). ${ }^{8,9}$ The double-contour sign of the hypoplastic medial trochlear facet on lateral knee radiographs is located posteriorly to the radiopaque line of the lateral trochlear facet (-Figs. 1, 5, and 6). ${ }^{8,9}$

Transverse CT or MR images in patients with trochlear dysplasia demonstrate a flattening or even an absent trochlear sulcus that might be assessed subjectively by the overall impression of the trochlear shape, by an trochlear sulcus angle $>145$ degrees, or a trochlear depth $\leq 3 \mathrm{~mm}$ on a transverse MR image $3 \mathrm{~cm}$ proximal to the femorotibial joint space. ${ }^{8,9,27}$ A marked ventral trochlear prominence $\geq 7 \mathrm{~mm}$ with a step-like transition zone between the anterior femoral cortex and the most ventral point of the trochlear floor on midsagittal MR images is an accurate criterion for trochlear dysplasia. ${ }^{27}$ A specific finding for trochlear dysplasia is a nipplelike anterior prominence at the most anterior and proximal part of the femoral trochlea on midsagittal MR images. ${ }^{27}$

True lateral radiographs revealed a higher diagnostic accuracy and are more reliable for the diagnosis of trochlear dysplasia compared with axial radiographs obtained at 30 degrees of flexion of the knee (-Figs. 2-7). ${ }^{26}$ True lateral radiographs allow assessment of the whole length of the trochlea. In contrast the evaluation of the trochlea on axial radiographs is limited to the small area viewed tangentially. ${ }^{26}$ The diagnostic accuracy of trochlear dysplasia is influenced by the degree of knee flexion. Axial views frequently enable radiologic assessment of the trochlea in its distal part, 


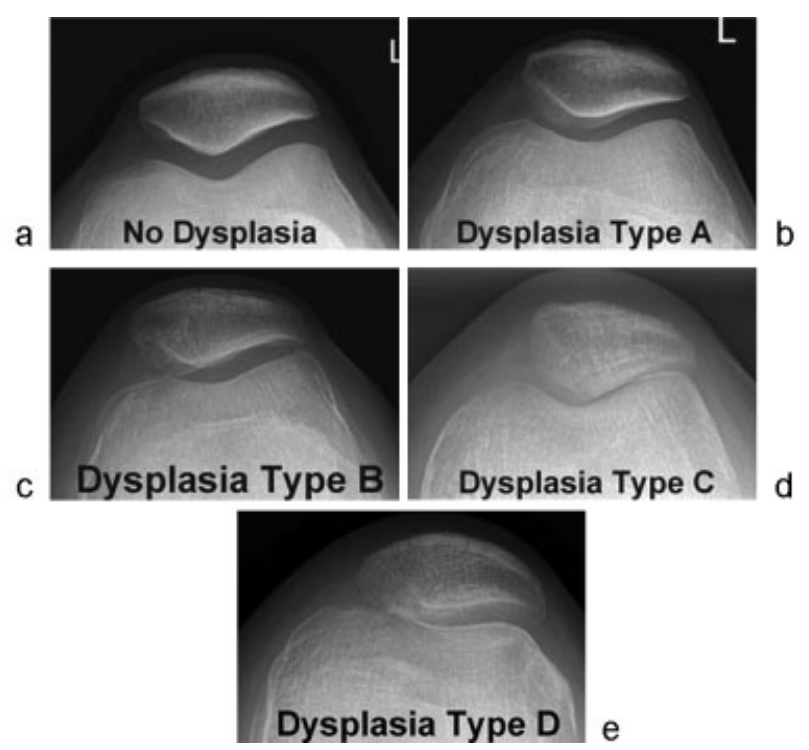

Fig. 7 (a-e) Normal trochlea and trochlear dysplasia types A, B, C, and $D$ on axial radiographs. This image series demonstrates the limited value of axial radiographs for the reliable diagnosis and classification of trochlear dysplasia. Thus diagnosis of trochlear dysplasia should be based on true lateral radiographs and transverse computed tomography or MR images. The radiographs are from the same patients as Figs. 2-6.

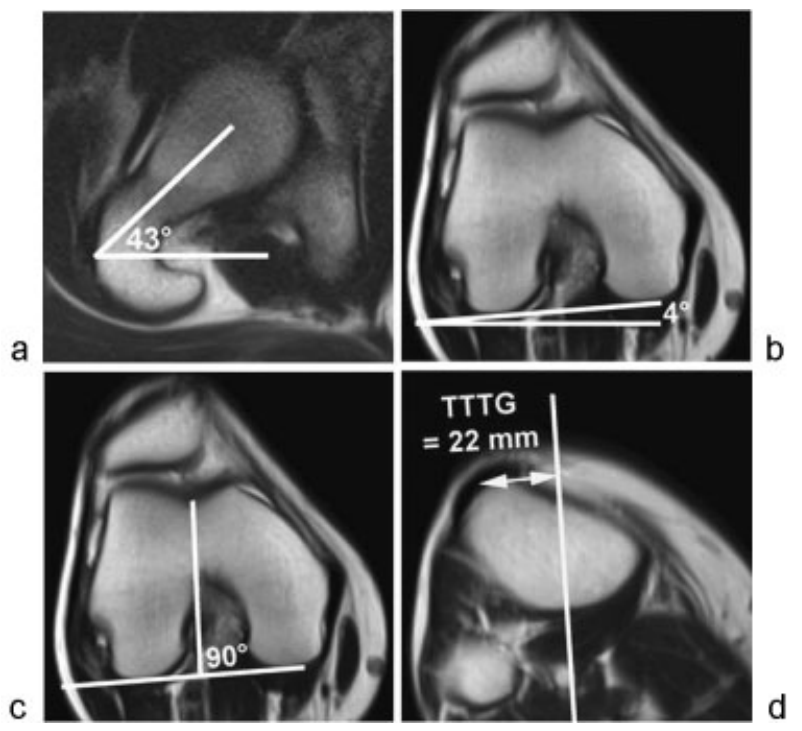

Fig. 8 Increased femoral antetorsion of 39 degrees (= 43 degrees on [a] minus 4 degrees on [b]) and increased tuberosity to the trochlear groove (TTTG) distance of $22 \mathrm{~mm}(\mathbf{c}, \mathbf{d})$. Femoral antetorsion and TTTG measurements are obtained in hip and knee extension as described in the text and references 37,74 , and 75 . These strictly transverse MR images over the proximal femur and knee are acquired by a fast imaging MR technique (single-shot turbo spin-echo) with the following parameters: femur: 25 sections per slab, $5 \mathrm{~mm}$ section thickness, 30\% intersection distance factor, $25 \mathrm{~s}$ acquisition time, spine matrix coil; knee: 18 sections per slab, $5 \mathrm{~mm}$ section thickness, $50 \%$ intersection distance factor, $25 \mathrm{~s}$ acquisition time, 18-channel body array flex coil.

whereas trochlea dysplasia is always located in the proximal part of the trochlea. ${ }^{26}$ Greater flexion angle of the knee on axial views delineate the more distal portion of the trochlea. ${ }^{9}$ Thus a maximum knee flexion of 45 degrees was suggested for axial conventional radiographs. ${ }^{9}$ Based on our experience, knee flexion angles $\leq 30$ degrees on axial views are difficult to realize due to a superimposition of the knee and feet in lower knee flexion angles.

An appropriate positioning to obtain a true lateral radiographs is crucial for the diagnostic accuracy of trochlear dysplasia. ${ }^{28}$ It was shown that a rotation deviation of 5 degrees of the distal femur can already cause false-positive or false-negative diagnosis of trochlear dysplasia in normal shaped and dysplastic femoral trochleae, respectively. ${ }^{28}$ Thus superimposition of the femoral condyles posteriorly is a good quality criterion for a true lateral radiograph and seems to be mandatory to diagnose and classify trochlear dysplasia correctly on lateral radiographs. ${ }^{28}$

Dejour's four-type trochlear dysplasia classification revealed a fair intraobserver and interobserver agreement on lateral knee radiographs and transverse MR images. ${ }^{29}$ The inter-method agreement for Dejour four-type trochlear dysplasia classification between lateral knee radiographs and transverse MR images was also only fair. ${ }^{29}$ It was also shown that lateral radiographs underestimate the degree of trochlear dysplasia according the Dejour classification compared with transverse MR images. ${ }^{29}$

Quantitative parameters such as the depth of the trochlear groove, condyle asymmetry, trochlear facet asymmetry, lateral trochlear inclination, lateralization of the patella, the height of the medial and lateral condyle, and the central trochlear height in relation to the width of the distal femur were evaluated on MR images to discriminate a normal and a dysplastic trochlea. ${ }^{30}$ The authors found that these quantitative measurements on MR images are of limited value for the assessment of trochlear dysplasia. ${ }^{30}$ In addition, measurements on transverse MR images of the femoral trochlea were not reliable to classify trochlear dysplasia type B, C, and D according to Dejour. ${ }^{30}$ The weak reliability in classification of trochlear dysplasia according to Dejour on radiographs and on CT and MR images suggest inconsistent treatment planning and a limited comparability of clinical outcomes in studies addressing trochlear dysplasia.

\section{Tuberosity to the Trochlear Groove Distance}

An increased lateralization of the tibial tubercle in relation to the trochlear groove lateralizes the force vector of the extensor mechanism of the knee. Lateralization of the tibial tubercle in relation to the trochlear groove can be quantified as the TTTG distance. The TTTG distance measurements were initially described on plain radiographs; today the TTTG distance is measured on CT and MR images. ${ }^{3,31,32}$ It was shown that an increased lateralized TTTG distance is an important risk factor in lateral patellar dislocations, which may be surgically corrected by an osteotomy and subsequent medial transfer of the tibial tubercle (-Figs. 1 and 8).,5,33-35 Conventional axial radiographs at 30 degrees of knee flexion revealed a large measurement error for the TTTG distance quantification compared with CT images. ${ }^{36}$ Thus conventional axial radiographs are not appropriate anymore for TTTG distance measurements. ${ }^{36}$ In contrast, cross-sectional modalities, either CT or MRI, are suitable for measurement of the TTTG distance ${ }^{32}$ : The following TTTG distance measurement technique is used 
at our institution: First, the most distal cross-sectional section with full cartilage coverage of the trochlear groove on transverse MR images or the equivalent level on CT images with continuous delineation of the trochlear cortex is selected. The cross-sectional image with the patellar tendon proximate to its insertion on the tibial tuberosity serves as the distal reference image. The TTTG is measured as the distance between the center of the patellar tendon at its tibial insertion and a reference line through the deepest point of the trochlear groove rectangular to the tangent along the posterior femoral condyles. ${ }^{37}$ TTTG distance measurements on CT and MR images revealed an excellent interobserver, intraobserver, and inter-method reliability. ${ }^{32,38,39}$ However, a broad range of TTTG threshold values between 15 and $20 \mathrm{~mm}$ or greater were proposed. ${ }^{3,5,33}$ This may be explained by some differences of the measurement techniques in the literature. ${ }^{37}$ For instance, TTTG values were acquired at various flexion and extension degrees of the knee; however, it was shown that the TTTG distance increases significantly in the end-stage extension of the knee. ${ }^{37,40,41}$ The mean TTTG values on MR images were $15.1 \pm 3.2 \mathrm{~mm}$ at full extension, $10.0 \pm 3.5 \mathrm{~mm}$ at 15 degrees flexion, and $8.1 \pm 3.4 \mathrm{~mm}$ at 30 degrees flexion in a well-balanced study cohort of asymptomatic volunteers. ${ }^{37}$ Using the MR body coil with the knee fully extended caused significantly higher TTTG values compared with MR examinations with a dedicated knee coil where the knee is slightly flexed. ${ }^{41}$ In addition, the dedicated knee coil often causes a slight varus alignment of the lower limb, which also explains in part the lower TTTG distances. ${ }^{39}$

As a consequence, the comparability of reported TTTG distance values obtained on radiographs, CT, and MRI at various flexion angles and varus alignment of the knee is limited. ${ }^{37,39}$ In particular, MRI in a dedicated knee coil may underestimate the TTTG distance compared with $\mathrm{CT}^{42}$

Anatomical variances and trochlear dysplasia also plays a crucial role in TTTG measurements: It was hypothesized that the reference point of the trochlear groove in trochlear dysplasia is more inferior lateral in comparison with normal trochlear grooves. ${ }^{43}$ As a consequence the TTTG distance is underestimated up to $3 \mathrm{~mm}$ in knees with trochlear dysplasia compared with normal knees. ${ }^{43}$ Furthermore, a decreasing inter- and intraobserver agreement was found with an increasing severity of trochlear dysplasia. ${ }^{44}$

The center of the patellar tendon at its tibial insertion as a soft tissue landmark is a more constant reference point and provides a higher interobserver and intraobserver agreement for TTTG measurements for surgical planning than its widely used osseous counterpart, the most anterior aspect of the tibial tubercle. ${ }^{45}$

\section{Patellar Tilt}

An easy and fast patellar tilt measurement on axial radiographs is the lateral patellofemoral angle, formed by a line between the femoral condyles anteriorly and a line between the margins of the lateral facet of the patella (- Fig. 9). ${ }^{46}$ The lateral patellofemoral angle was open laterally in $97 \%$ and with parallel lines in $3 \%$ of 100 normal control patients on axial radiographs with a

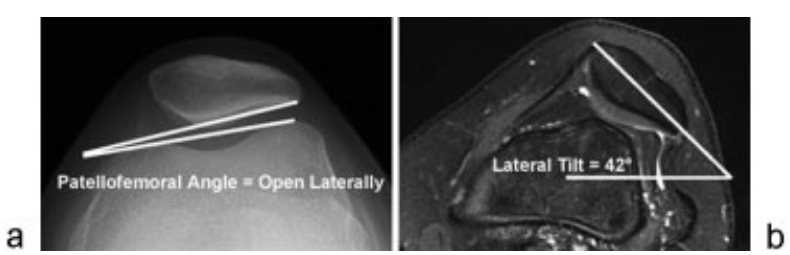

Fig. 9 Lateral patellar tilt on a radiograph compared with MRI. Normal patellofemoral angle without pathologic lateral tilt on an axial radiograph at a knee flexion of 40 degrees. Severely increased lateral tilt on the MRI obtained at a slight knee flexion. The lateral patellar tilt is influenced by various flexion angles of the knee )reference 40, 49, and 57). The lateral patellar tilt is currently not considered an independent anatomical risk factor for patellar instability.

knee flexion between 20 and 30 degrees. In contrast, the lateral patellofemoral angle ${ }^{46}$ was open medially in $20 \%$ and with parallel lines in $80 \%$ of patients with recurrent symptomatic patellar subluxations. $^{46}$

On transverse CT images, a reference line through the transverse axis of the patella and a line tangential along the femoral condyles posteriorly determine the lateral patellar tilt angle (-Fig. 9). ${ }^{3,8}$ The mean lateral patellar tilt angle on transverse CT with the knee in extension without quadriceps contraction was significantly higher in patients with patellar instability (28.8 \pm 10.5 degrees) compared with the control group (10 \pm 5.8 degrees). Quadriceps contraction increased the lateral patellar tilt by a mean of 6 degrees in patients with patellar instability, whereas the control group demonstrated an increase of 1.5 degrees. The authors suggested a threshold value of 20 degrees for lateral patellar tilt on transverse CT images with the knee in extension without quadriceps contraction. Similar lateral patellar tilt characteristics on transverse $\mathrm{CT}$ images with and without quadriceps contraction were noted in further studies. ${ }^{47,48}$

Thus prominent lateral patellar tilt was assessed as a major anatomical risk factor for patellar instability. ${ }^{3,8,47}$ Extensor apparatus insufficiency, in particular quadriceps dysplasia and vastus medialis dysplasia, was considered as the etiologic factor of prominent lateral tilt in the past. ${ }^{3,8,47}$

However, the current literature has shown that increased lateral patellar tilt is rather a consequence of an increased TTTG distance, patella alta, trochlear dysplasia, insufficient MPFL, and various flexion angles of the femorotibial compartments than an independent anatomical risk factor for patellar instability. ${ }^{40,49-57}$ In addition it was hypothesized ${ }^{58}$ that a severe knee effusion could increase or diminish patellar tilt.

Finally, it has to be noted that a meta-analysis revealed insufficient evidence to determine the reliability, validity, sensitivity, or specificity of lateral patellar tilt as a radiologic assessment parameter for patellar instability. ${ }^{59}$

\section{Medial Patellofemoral Ligament}

The MPFL is consistently found in anatomical dissection with a mean width of $1.9 \mathrm{~cm}$ and a mean length of $5.3 \mathrm{~cm} .{ }^{60}$ Most of the MPFL fibers originate close to the adductor tubercle of the femur, distal to the attachment of the adductor magnus tendon and proximal to the origin of the medial collateral ligament. ${ }^{60}$ The 
MPFL attaches at the proximal two thirds of the medial margin of the patella. ${ }^{60} \mathrm{~A}$ broad range in thickness and quality of the MPFL was found. ${ }^{60}$ The MPFL has a close anatomical relationship with contributing fibers from and to the posteromedial joint capsule, the medial collateral ligament, the medial patellar retinaculum as well as the quadriceps fibers, in particular the musculus vastus medialis obliquus and the vastus intermedius. ${ }^{5,60}$ Thus the femoral origin of the MPFL and the free distal edge of the MPFL frequently cannot be clearly delineated in anatomical dissections. ${ }^{60,61}$ In addition, the medial patellar retinaculum and the MPFL blend at their patellar insertion and are often difficult to differentiate from each other on routine MR images. ${ }^{5}$

The MPFL is the main medial passive stabilizer of the patellofemoral joint at full extension of the knee and slight flexion up to 20 degrees. $^{2,61}$ A biomechanical study has shown that the required force reduced by $\sim 50 \%$ to displace the patella laterally in a fully extended knee with a torn MPFL compared with a knee with an intact MPFL. ${ }^{2}$ This main patellar-stabilizing function of the MPFL decreased promptly as the knee flexed. ${ }^{2}$

Tears of the MPFL may occur in its midportion, at its femoral or patellar attachment, or as combined MPFL tears with several injured ligamentous parts. There are controversial data about the most frequent location of a tear of the MPFL. Some studies found more MPFL tears at the femoral origin compared with the patellar attachment after lateral patellar dislocation and vice versa. ${ }^{62-68}$ It was found that the various MPFL injury patterns are related to trochlear dysplasia, patellar height, and TTTG distance. ${ }^{64}$

It was hypothesized that insufficiency to the MPFL is a precondition for an abnormal lateral patellar movement such as in lateral patellar dislocation. ${ }^{61}$ The frequency of a partial or completely torn medial patellofemoral ligaments after acute lateral patellar dislocation varies between $78 \%$ and $100 \%$; however, most studies reported an injured MPFL in $>90 \% .{ }^{62-66,68}$ The very high pretest probability of $\sim 90 \%$ of a partial or completely torn MPFL in patients with a history, clinical and imaging findings of an previous acute lateral patellar dislocations suggests that the therapeutic concept of the orthopedic surgeon in patellar instability will be rather influenced by the locally preferred standard indication regarding MPFL reconstruction than by the MRI findings of the MPFL.

\section{Torsional Limb Alignment}

Thirty patients with a history of recent patellar dislocation demonstrated significant higher mean values compared with asymptomatic volunteers for femoral antetorsion $(20.3 \pm 10.4$ degrees versus $13.0 \pm 8.4$ degrees), knee rotation (9.4 \pm 5.0 degrees versus $5.7^{\circ} \pm 4.3$ degrees) and genu valgum $(0.81 \pm 0.75 \mathrm{~mm}$ versus $-0.28 \pm 0.87 \mathrm{~mm})$ on MR images. ${ }^{11}$ The corresponding tibial torsion values did not differ significantly. ${ }^{11}$ In addition, quantitative values for trochlear dysplasia, patellar height, and TTTG distance served for the assessment of standard anatomical risk factors for patellar instability and were compared with the torsional alignment values. ${ }^{11}$ These standard anatomical risk factors did not correlate significantly with the obtained torsional alignment parameters. ${ }^{11}$ The authors concluded that torsional malalignment might be a so far underestimated primary risk factor in patellar dislocation. ${ }^{11}$

\section{Gender-Specific Differences}

Females, in particular between 10 and 17 years of age, have a higher risk for patellofemoral instability. ${ }^{69}$ A 33\% higher frequency for acute patellar dislocations was reported in female compared with male adolescents with a three times higher risk for recurrent patellar dislocations. ${ }^{34,69}$ The higher frequency for patellofemoral instability in females compared with males diminishes by increasing age; however, a slight female predominance persist in adults with a women-tomen-ratio of 54 to $46 \%$ for first-time patellar dislocations. $^{34,69,70}$ Balcarek et al $^{34}$ analyzed the distribution of no-risk pivoting activities (e.g., swimming, cycling), lowrisk pivoting activities (e.g., hiking, jogging, dancing), and high-risk pivoting activities (e.g., football, basketball, soccer) for first-time patellar dislocations and found significant gender differences. The authors reported that low-risk and no-risk pivoting activities were more frequent in females with first-time patellar dislocations, whereas high-risk pivoting activities were more common in males. The authors also found that trochlear dysplasia and the TTTG distance is more pronounced in women who dislocate the patella compared with men. ${ }^{34}$ Thus it was concluded that the more excessive trochlear dysplasia and the higher TTTG distance in female patients with patellar instability might contribute to an increased risk of lateral patellar dislocations for females compared with males. ${ }^{34}$

In contrast, the TTTG distance and the patellar height did not differ significantly between males and females in the overall study population of Balcarek et al consisting of 100 patients with lateral patellar instability and 157 patients without patellar instability $(p$ value $=0.94) .{ }^{34}$

\section{Current Therapeutic Concepts at Our Institution}

Patellar instability is multifactorial; thus imaging and therapeutic strategies have to address several anatomical variants in patients with lateral dislocations of the patella. $3,7,10,14,71$ Conservative and operative treatment strategies for patellar instability are highly individual. ${ }^{7}$

Recurrent symptomatic patellar dislocations are much more frequent in patients with previous multiple patellar instability episodes (49\%) compared with first-time patellar dislocation patients (17\%). ${ }^{69}$ Thus a conservative therapy is usually chosen for first-time patellar dislocation patients at our institution. ${ }^{7}$ Conservative treatment includes physiotherapy, bracing, weight reduction, and pain medication. ${ }^{7,71}$

In contrast, recurrent lateral patellar dislocations usually undergo surgical interventions such as MPFL reconstruction, sulcus-deepening trochleoplasty, and distalization and medialization of the tibial tubercle. ${ }^{7,14}$ Lateral retinaculum release or lengthening is an uncommon procedure for cases with patellar tilting and the clinically diagnosed severe lateral patellar 
compression syndrome., ${ }^{74,72}$ The indication for femoral derotation osteotomy is reserved for rare occasions. ${ }^{7,14}$

MPFL reconstruction is performed in most of the patients with recurrent patellar dislocations. ${ }^{7}$ Distalization of the tibial tubercle is done in patients with prominent patella alta with a Caton-Deschamps index $>1.3$ with a goal index close to 1.0. Medialization of the tibial tubercle is indicated for a TTTG distance $\geq 20 \mathrm{~mm}$. ${ }^{3,7,73}$ A TTTG distance $\sim 12 \mathrm{~mm}$ should be achieved postoperatively.

Trochlear dysplasia severe type B and type D have showed a significantly better subjective outcome compared with type A and mild type $\mathrm{B}^{14}$ Thus a stricter indication for trochleoplasty was accepted at our institution than is commonly indicated for type D and severe type B trochlear dysplasia and usually not for type $A$ and mild type $B{ }^{14}$ Type $C$ can be treated by trochleoplasty in cases with relevant dysmorphic changes. Trochleoplasty also lateralizes the sulcus, which indirectly corrects an increased TTTG distance by lowering the lateral vector force of the patellar tendon. ${ }^{14}$ Thus additional medialization osteotomy of the tibial tubercle is less frequently indicated in trochleoplasty patients. ${ }^{14}$

\section{Summary}

The most important imaging examinations for the work-up of patients with patellar instability are the true lateral radiograph and transverse CT or MR images of the knee. The pretest likelihood of a torn MPFL on MR images is very high after a lateral patellar dislocation. Increased lateral patellar tilt is rather a consequence of primary anatomical risk factors than an independent anatomical risk factor for patellar instability. Radiologic reports should provide details about the individual and independent main anatomical risk factors for patellar instability such as patella alta, trochlear dysplasia, increased TTTG distance, and torsional limb parameters.

\section{References}

1 Koh JL, Stewart C. Patellar instability. Clin Sports Med 2014;33(3): 461-476

2 Senavongse W, Amis AA. The effects of articular, retinacular, or muscular deficiencies on patellofemoral joint stability: a biomechanical study in vitro. J Bone Joint Surg Br 2005;87(4): 577-582

3 Dejour H, Walch G, Nove-Josserand L, Guier C. Factors of patellar instability: an anatomic radiographic study. Knee Surg Sports Traumatol Arthrosc 1994;2(1):19-26

4 Sherman SL, Plackis AC, Nuelle CW. Patellofemoral anatomy and biomechanics. Clin Sports Med 2014;33(3):389-401

5 Diederichs G, Issever AS, Scheffler S. MR imaging of patellar instability: injury patterns and assessment of risk factors. Radiographics 2010;30(4):961-981

6 Dejour DH. The patellofemoral joint and its historical roots: the Lyon School of Knee Surgery. Knee Surg Sports Traumatol Arthrosc 2013;21(7):1482-1494

7 M Tscholl P, P Koch P, F Fucentese S. Treatment options for patellofemoral instability in sports traumatology. Orthop Rev (Pavia) 2013;5(3):e23

8 Dejour D, Le Coultre B. Osteotomies in patello-femoral instabilities. Sports Med Arthrosc Rev 2007;15(1):39-46
9 Dejour D, Saggin P. The sulcus deepening trochleoplasty-the Lyon's procedure. Int Orthop 2010;34(2):311-316

10 Steensen RN, Bentley JC, Trinh TQ, Backes JR, Wiltfong RE. The prevalence and combined prevalences of anatomic factors associated with recurrent patellar dislocation: a magnetic resonance imaging study. Am J Sports Med 2015;43(4):921-927

11 Diederichs G, Köhlitz T, Kornaropoulos E, Heller MO, Vollnberg B, Scheffler S. Magnetic resonance imaging analysis of rotational alignment in patients with patellar dislocations. Am J Sports Med 2013;41(1):51-57

12 Gheno R, Nectoux E, Herbaux B, et al. Three-dimensional measurements of the lower extremity in children and adolescents using a low-dose biplanar X-ray device. Eur Radiol 2012;22(4):765-771

13 Caton JH, Dejour D. Tibial tubercle osteotomy in patello-femoral instability and in patellar height abnormality. Int Orthop 2010; 34(2):305-309

14 Fucentese SF, Zingg PO, Schmitt J, Pfirrmann CW, Meyer DC, Koch PP. Classification of trochlear dysplasia as predictor of clinical outcome after trochleoplasty. Knee Surg Sports Traumatol Arthrosc 2011;19(10):1655-1661

15 Amis AA, Senavongse W, Bull AM. Patellofemoral kinematics during knee flexion-extension: an in vitro study. J Orthop Res 2006;24(12):2201-2211

16 Grelsamer RP, Meadows S. The modified Insall-Salvati ratio for assessment of patellar height. Clin Orthop Relat Res 1992;(282): 170-176

17 Caton J, Deschamps G, Chambat P, Lerat JL, Dejour H. Patella infera. Apropos of 128 cases [in French]. Rev Chir Orthop Repar Appar Mot 1982;68(5):317-325

18 Blackburne JS, Peel TE. A new method of measuring patellar height. J Bone Joint Surg Br 1977;59(2):241-242

19 Insall J, Salvati E. Patella position in the normal knee joint. Radiology 1971;101(1):101-104

20 Nizić D, Pervan M, Kovačević B. A new reference line in diagnosing a high-riding patella on routine digital lateral radiographs of the knee. Skeletal Radiol 2014;43(8):1129-1137

21 Phillips CL, Silver DA, Schranz PJ, Mandalia V. The measurement of patellar height: a review of the methods of imaging. J Bone Joint Surg Br 2010;92(8):1045-1053

22 Aparicio G, Abril JC, Albiñana J, Rodríguez-Salvanés F. Patellar height ratios in children: an interobserver study of three methods. J Pediatr Orthop B 1999;8(1):29-32

23 Thévenin-Lemoine C, Ferrand M, Courvoisier A, Damsin JP, Ducou le Pointe $\mathrm{H}$, Vialle $\mathrm{R}$. Is the Caton-Deschamps index a valuable ratio to investigate patellar height in children? J Bone Joint Surg Am 2011;93(8):e35

24 Miller TT, Staron RB, Feldman F. Patellar height on sagittal MR imaging of the knee. AJR Am J Roentgenol 1996;167(2):339-341

25 Lee PP, Chalian M, Carrino JA, Eng J, Chhabra A. Multimodality correlations of patellar height measurement on X-ray, CT, and MRI. Skeletal Radiol 2012;41(10):1309-1314

26 Malghem J, Maldague B. Depth insufficiency of the proximal trochlear groove on lateral radiographs of the knee: relation to patellar dislocation. Radiology 1989;170(2):507-510

27 Pfirrmann CW, Zanetti M, Romero J, Hodler J. Femoral trochlear dysplasia: MR findings. Radiology 2000;216(3):858-864

28 Koëter S, Bongers EM, de Rooij J, van Kampen A. Minimal rotation aberrations cause radiographic misdiagnosis of trochlear dysplasia. Knee Surg Sports Traumatol Arthrosc 2006;14(8):713-717

29 Lippacher S, Dejour D, Elsharkawi M, et al. Observer agreement on the Dejour trochlear dysplasia classification: a comparison of true lateral radiographs and axial magnetic resonance images. Am J Sports Med 2012;40(4):837-843

30 Nelitz M, Lippacher S, Reichel H, Dornacher D. Evaluation of trochlear dysplasia using MRI: correlation between the classification system of Dejour and objective parameters of trochlear dysplasia. Knee Surg Sports Traumatol Arthrosc 2014;22(1):120-127 
31 Goutallier D, Bernageau J, Lecudonnec B. The measurement of the tibial tuberosity. Patella groove distanced technique and results (author's transl) [in French]. Rev Chir Orthop Repar Appar Mot 1978;64(5):423-428

32 Schoettle PB, Zanetti M, Seifert B, Pfirrmann CW, Fucentese SF, Romero J. The tibial tuberosity-trochlear groove distance; a comparative study between CT and MRI scanning. Knee 2006;13(1):26-31

33 Koëter S, Diks MJ, Anderson PG, Wymenga AB. A modified tibial tubercle osteotomy for patellar maltracking: results at two years. J Bone Joint Surg Br 2007;89(2):180-185

34 Balcarek P, Jung K, Ammon J, et al. Anatomy of lateral patellar instability: trochlear dysplasia and tibial tubercle-trochlear groove distance is more pronounced in women who dislocate the patella. Am J Sports Med 2010;38(11):2320-2327

35 Dickens AJ, Morrell NT, Doering A, Tandberg D, Treme G. Tibial tubercle-trochlear groove distance: defining normal in a pediatric population. J Bone Joint Surg Am 2014;96(4):318-324

36 Wagenaar FC, Koëter S, Anderson PG, Wymenga AB. Conventional radiography cannot replace $C T$ scanning in detecting tibial tubercle lateralisation. Knee 2007;14(1):51-54

37 Dietrich TJ, Betz M, Pfirrmann CW, Koch PP, Fucentese SF. Endstage extension of the knee and its influence on tibial tuberositytrochlear groove distance (TTTG) in asymptomatic volunteers. Knee Surg Sports Traumatol Arthrosc 2014;22(1):214-218

38 Skelley N, Friedman M, McGinnis M, Smith C, Hillen T, Matava M. Inter- and intraobserver reliability in the MRI measurement of the tibial tubercle-trochlear groove distance and trochlea dysplasia. Am J Sports Med 2015;43(4):873-878

39 Ho CP, James EW, Surowiec RK, et al. Systematic techniquedependent differences in CT versus MRI measurement of the tibial tubercle-trochlear groove distance. Am J Sports Med 2015;43(3): 675-682

40 Becher C, Fleischer B, Rase M, et al. Effects of upright weight bearing and the knee flexion angle on patellofemoral indices using magnetic resonance imaging in patients with patellofemoral instability. Knee Surg Sports Traumatol Arthrosc 2015; October 19 (Epub ahead of print)

41 Aarvold A, Pope A, Sakthivel VK, Ayer RV. MRI performed on dedicated knee coils is inaccurate for the measurement of tibial tubercle trochlear groove distance. Skeletal Radiol 2014;43(3): 345-349

42 Camp CL, Stuart MJ, Krych AJ, et al. CT and MRI measurements of tibial tubercle-trochlear groove distances are not equivalent in patients with patellar instability. Am J Sports Med 2013;41(8): 1835-1840

43 Tscholl PM, Antoniadis A, Dietrich TJ, Koch PP, Fucentese SF. The tibial-tubercle trochlear groove distance in patients with trochlear dysplasia: the influence of the proximally flat trochlea. Knee Surg Sports Traumatol Arthrosc 2014; October 19 (Epub ahead of print)

44 Dornacher D, Reichel H, Lippacher S. Measurement of tibial tuberosity-trochlear groove distance: evaluation of inter- and intraobserver correlation dependent on the severity of trochlear dysplasia. Knee Surg Sports Traumatol Arthrosc 2014;22(10): 2382-2387

45 Wilcox JJ, Snow BJ, Aoki SK, Hung M, Burks RT. Does landmark selection affect the reliability of tibial tubercle-trochlear groove measurements using MRI? Clin Orthop Relat Res 2012;470(8): 2253-2260

46 Laurin CA, Lévesque HP, Dussault R, Labelle H, Peides JP. The abnormal lateral patellofemoral angle: a diagnostic roentgenographic sign of recurrent patellar subluxation. J Bone Joint Surg Am 1978;60(1):55-60

47 Nove-Josserand L, Dejour D. Quadriceps dysplasia and patellar tilt in objective patellar instability [in French]. Rev Chir Orthop Repar Appar Mot 1995;81(6):497-504

48 Schueda MA, Astur DC, Bier RS, Bier DS, Astur N, Cohen M. Use of computed tomography to determine the risk of patellar dislocation in 921 patients with patellar instability. Open Access J Sports Med 2015;6:55-62

49 Tanaka MJ, Elias JJ, Williams AA, Carrino JA, Cosgarea AJ. Correlation between changes in tibial tuberosity-trochlear groove distance and patellar position during active knee extension on dynamic kinematic computed tomographic imaging. Arthroscopy 2015;31(9):1748-1755

50 Stephen JM, Dodds AL, Lumpaopong P, Kader D, Williams A, Amis AA. The ability of medial patellofemoral ligament reconstruction to correct patellar kinematics and contact mechanics in the presence of a lateralized tibial tubercle. Am J Sports Med 2015; 43(9):2198-2207

51 Pal S, Besier TF, Beaupre GS, Fredericson M, Delp SL, Gold GE. Patellar maltracking is prevalent among patellofemoral pain subjects with patella alta: an upright, weightbearing MRI study J Orthop Res 2013;31(3):448-457

52 Ward SR, Terk MR, Powers CM. Patella alta: association with patellofemoral alignment and changes in contact area during weight-bearing. J Bone Joint Surg Am 2007;89(8): 1749-1755

53 Powers CM. Patellar kinematics, part II: the influence of the depth of the trochlear groove in subjects with and without patellofemoral pain. Phys Ther 2000;80(10):965-978

54 Van Haver A, De Roo K, De Beule M, et al. The effect of trochlear dysplasia on patellofemoral biomechanics: a cadaveric study with simulated trochlear deformities. Am J Sports Med 2015;43(6): 1354-1361

55 Schöttle PB, Fucentese SF, Romero J. Clinical and radiological outcome of medial patellofemoral ligament reconstruction with a semitendinosus autograft for patella instability. Knee Surg Sports Traumatol Arthrosc 2005;13(7):516-521

56 Wagner D, Pfalzer F, Hingelbaum S, Huth J, Mauch F, Bauer G. The influence of risk factors on clinical outcomes following anatomical medial patellofemoral ligament (MPFL) reconstruction using the gracilis tendon. Knee Surg Sports Traumatol Arthrosc 2013;21(2): 318-324

57 Zaffagnini S, Colle F, Lopomo N, et al. The influence of medial patellofemoral ligament on patellofemoral joint kinematics and patellar stability. Knee Surg Sports Traumatol Arthrosc 2013; 21(9):2164-2171

58 Grelsamer RP, Weinstein CH, Gould J, Dubey A. Patellar tilt: the physical examination correlates with MR imaging. Knee 2008; 15(1):3-8

59 Smith TO, Davies L, Toms AP, Hing CB, Donell ST. The reliability and validity of radiological assessment for patellar instability. A systematic review and meta-analysis. Skeletal Radiol 2011; 40(4):399-414

60 Tuxøe JI, Teir M, Winge S, Nielsen PL. The medial patellofemoral ligament: a dissection study. Knee Surg Sports Traumatol Arthrosc 2002;10(3):138-140

61 Amis AA, Firer P, Mountney J, Senavongse W, Thomas NP. Anatomy and biomechanics of the medial patellofemoral ligament. Knee 2003;10(3):215-220

62 Elias DA, White LM, Fithian DC. Acute lateral patellar dislocation at MR imaging: injury patterns of medial patellar soft-tissue restraints and osteochondral injuries of the inferomedial patella. Radiology 2002;225(3):736-743

63 Guerrero P, Li X, Patel K, Brown M, Busconi B. Medial patellofemoral ligament injury patterns and associated pathology in lateral patella dislocation: an MRI study. Sports Med Arthrosc Rehabil Ther Technol 2009;1(1):17

64 Balcarek P, Ammon J, Frosch S, et al. Magnetic resonance imaging characteristics of the medial patellofemoral ligament lesion in acute lateral patellar dislocations considering trochlear dysplasia, patella alta, and tibial tuberosity-trochlear groove distance. Arthroscopy 2010;26(7):926-935

65 Seeley M, Bowman KF, Walsh C, Sabb BJ, Vanderhave KL. Magnetic resonance imaging of acute patellar dislocation in children: 
patterns of injury and risk factors for recurrence. J Pediatr Orthop 2012;32(2):145-155

66 Balcarek P, Walde TA, Frosch S, et al. Patellar dislocations in children, adolescents and adults: a comparative MRI study of medial patellofemoral ligament injury patterns and trochlear groove anatomy. Eur J Radiol 2011;79(3):415-420

67 Sillanpää PJ, Peltola E, Mattila VM, Kiuru M, Visuri T, Pihlajamäki H. Femoral avulsion of the medial patellofemoral ligament after primary traumatic patellar dislocation predicts subsequent instability in men: a mean 7-year nonoperative follow-up study. Am J Sports Med 2009;37(8):1513-1521

68 Sanders TG, Morrison WB, Singleton BA, Miller MD, Cornum KG. Medial patellofemoral ligament injury following acute transient dislocation of the patella: MR findings with surgical correlation in 14 patients. J Comput Assist Tomogr 2001;25(6):957-962

69 Fithian DC, Paxton EW, Stone ML, et al. Epidemiology and natural history of acute patellar dislocation. Am J Sports Med 2004;32(5): 1114-1121
70 Stefancin JJ, Parker RD. First-time traumatic patellar dislocation: a systematic review. Clin Orthop Relat Res 2007;455(455): 93-101

71 Smith TO, Donell S, Song F, Hing CB. Surgical versus non-surgical interventions for treating patellar dislocation. Cochrane Database Syst Rev 2015;2(2):CD008106

72 Saper MG, Shneider DA. Diagnosis and treatment of lateral patellar compression syndrome. Arthrosc Tech 2014;3(5):e633-e638

73 Colvin AC, West RV. Patellar instability. J Bone Joint Surg Am 2008; 90(12):2751-2762

74 Sutter R, Dietrich TJ, Zingg PO, Pfirrmann CW. Femoral antetorsion: comparing asymptomatic volunteers and patients with femoroacetabular impingement. Radiology 2012;263(2): 475-483

75 Sutter R, Dietrich TJ, Zingg PO, Pfirrmann CW. Assessment of femoral antetorsion with MRI: comparison of oblique measurements to standard transverse measurements. AJR Am J Roentgenol 2015;205(1):130-135 